\title{
Energy demand in sludge dewatering
}

\author{
C.P. Chu ${ }^{\mathrm{a}}$, D.J. Lee ${ }^{\mathrm{a}, *}$, C.Y. Chang ${ }^{\mathrm{b}}$ \\ ${ }^{a}$ Department of Chemical Engineering, National Taiwan University, 1, Sec. 4 Roosevelt Road, Taipei, Taiwan, 10617, ROC \\ ${ }^{\mathrm{b}}$ Graduate Institute of Environmental Engineering, National Taiwan University, Taipei, Taiwan, 10617, ROC
}

Received 26 April 2004; received in revised form 12 December 2004; accepted 9 February 2005

Available online 18 April 2005

\begin{abstract}
This work investigates the energy required to dewater a suspension, i.e., activated sludge dewatered by centrifugation or consolidation. Total energy input to the suspension from the dewatering device, bond strength between adjacent water and solid surface, and intra-cake friction loss were evaluated for original and flocculated sludges. In centrifugal dewatering, most energy input during the initial stage was consumed by overcoming process irreversibility other than intra-cake friction, and, thereby, had a low energy efficiency. To increase centrifuge speed or to flocculate the sludge at optimal flocculant dosage would yield a high-energy input. In the consolidation test, most energy input at the initial stage was consumed in breaking down the bond strength until the moisture content reduced to less than the critical content. During subsequent dewatering stages, friction loss became the dominant source of energy loss. Dewatering sludge with high-energy efficiency is beneficial to optimally operate a dewatering process.
\end{abstract}

(C) 2005 Elsevier Ltd. All rights reserved.

Keywords: Energy; Efficiency; Bond strength; Friction loss; Centrifugation; Consolidation; Flocculation

\section{Introduction}

Energy consumption rate is an essential parameter determining the economy of dewatering a suspension by applying a solid-liquid separation (SLS) device. Professionals acquired knowledge by experience regarding energy cost for specific SLS processes. The minimum energy required to achieve a dewatering operation is both of academic and practical interest since the extent to which a given process deviates from an ideal dewatering operation can be estimated. To reduce the process irreversibility provides ways of maximizing energy efficiency (Bejan, 1996). Restated, if the ratio of real energy input to minimum energy requirement defined energy efficiency, then the process dewatering

\footnotetext{
*Corresponding author. Tel.: +886223625632; fax: +886223623040 .

E-mail address: djlee@ntu.edu.tw (D.J. Lee).
}

performance could be evaluated based on energy economy aspect.

Water with high water-to-solid bond strength can be referred to as sludge "bound water" (Vesilind, 1994). Various methods have been proposed for measuring bound water content (Heukelekian and Weisberg, 1956; Lee et al., 1975; Haschemeyer et al., 1977; Lewicki et al., 1978; Karr and Keinath, 1978; Katsiris and KouzeliKatsiri, 1987; Herwijn et al., 1992; Robinson and Knocke, 1992; Lee and Hsu, 1995). Kopp and Dichtl (2000, 2001a, b) proposed the correlation between the moisture distribution in sludge and the efficiency of sludge dewatering in full-scale plant. Chen et al. (1997, 1999) proposed a continuous scheme that considers the water-solid bond strength as a physically relevant classification index to assess the status of water in sludge. Driving "freed" water out of the suspension requires sufficient energy to overcome irreversible internal processes, such as cake and filter media fluid 


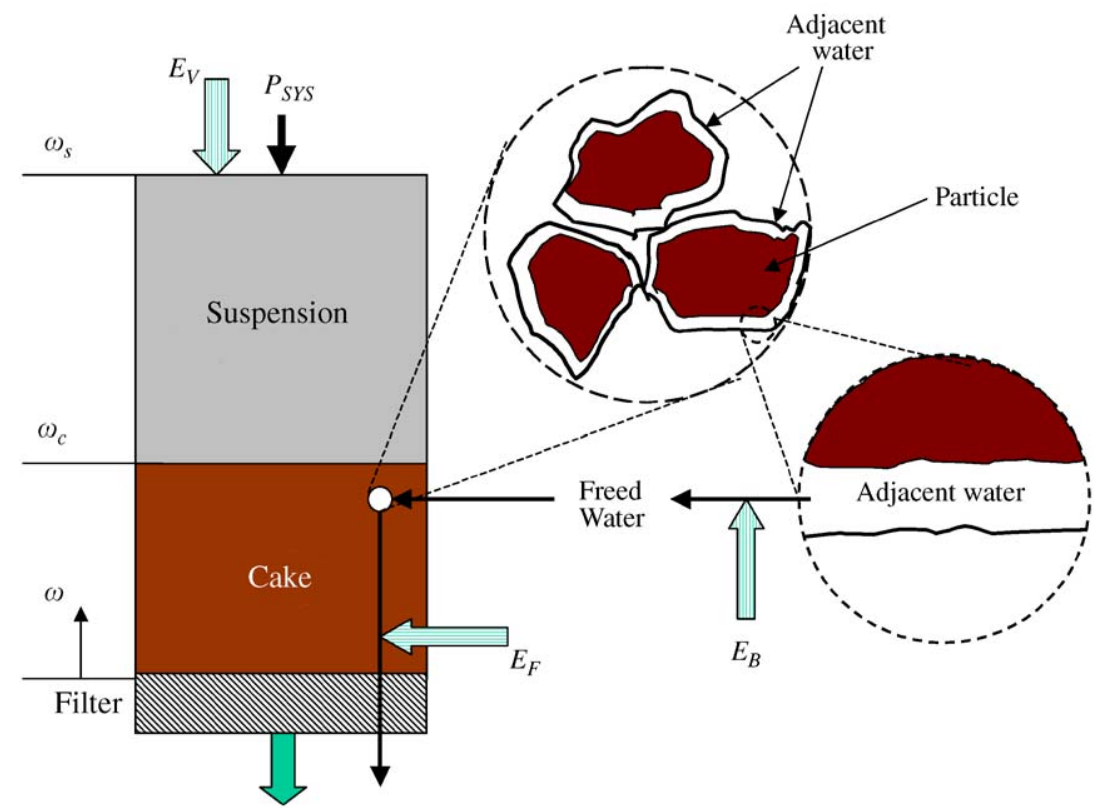

Filtrate

Fig. 1. The schematics of the moisture removal process. $E_{\mathrm{V}}: p-V$ work done by the surroundings, $E_{\mathrm{B}}$ : energy needed to release the adjacent water from the bound surface; $E_{\mathrm{F}}$ : energy required to overcome the friction loss of water flow through the cake and the filter.

friction. Modified Darcy's law is typically used to describe this friction force (Lee and Wang, 2000). The sum of the bond strength and associated friction loss yielded the theoretically minimum amount of work needed to dewater a suspension, $t$.

Removing water from a suspension requires that (1) the bond strength between the adjacent water and the solid surface are broken down, without physically moving the water from its original position near the solid surface; and (2) that this water is moved from its original position in suspension to outside the system through the sludge cake and the filter medium. The energy needed to separate the adjacent water from the solid surface is denoted by $E_{\mathrm{B}}$, and the energy required to force this water through the filter cake and filter medium is $E_{\mathrm{F}}$. Therefore, the minimum energy requirement to dewater a sludge is $\left(E_{\mathrm{B}}+E_{\mathrm{F}}\right)$. If the energy consumed by the dewatering device $\left(E_{\mathrm{V}}\right)$ is higher than this minimum energy requirement, and the difference is $\left(E_{\mathrm{V}}-\left(E_{\mathrm{B}}+E_{\mathrm{F}}\right)\right)$, presented the irreversibility yielded by other process non-ideality. This work estimated the minimum energy needed to dewater a suspension, and used an activated sludge as the test example. Bond strength of water $\left(E_{\mathrm{B}}\right)$ was estimated by utilizing the method proposed by Chen et al. (1997). Then, pressure and volume work done on the suspension $\left(E_{\mathrm{V}}\right)$ and friction loss during the consolidated dewatering or centrifugal dewatering $\left(E_{\mathrm{F}}\right)$ were theoretically derived and evaluated based on experimental data. The removal process of moisture in suspension was schematically shown in Fig. 1. In this figure, $\omega$ is the solid volume coordinate per unit area of filter media, including cake, sediment, and supernatant, and where $\omega_{\mathrm{s}}$ is the total solid volume above unit filter medium.

\section{Energy demand in dewatering}

\subsection{Energy input}

A mechanical dewatering device removes water from a suspension by supplying energy at a power level $P$. Consider that only some of the energy input is adopted during dewatering, characterized by an average efficiency of $\eta_{\text {ave }}$, then the input energy can be expressed as

$E_{\mathrm{V}}=\eta_{\text {ave }} \int_{0}^{t} P \mathrm{~d} t$,

where $E_{\mathrm{V}}$ is total energy input received by sludge, and $t$ is the dewatering time.

The mechanical work received by the sludge (solids and water that remain in the dewatering device) from the external environment is attributable to the change in volume of the sludge under shear owing to the removal of filtrate (Herwijn et al., 1992):

$$
\begin{aligned}
\mathrm{d} E_{\mathrm{V}} & =\left(p_{\text {SYS }}+\mathrm{d} p_{\text {SYS }}\right)(V+\mathrm{d} V)-p_{\text {SYS }} V, \\
& =p_{\text {SYS }} \mathrm{d} V+V \mathrm{~d} p_{\text {SYS }},
\end{aligned}
$$


where $p_{\mathrm{SYS}}$ is the pressure exerted by the surroundings to the sludge. Therefore, based on the solid volume unit area of the filter, pressure-volume work can be expressed as

$$
\begin{aligned}
\frac{\partial E_{\mathrm{V}}}{\partial \omega}= & H_{\mathrm{V}}=p_{\mathrm{SYS}} \frac{\partial V}{\partial \omega}+V \frac{\partial p_{\mathrm{SYS}}}{\partial \omega}, \\
= & p_{\mathrm{SYS}}(\omega, t) \frac{\partial\left\{V_{\mathrm{S}}[1+e(\omega, t)]\right\}}{\partial \omega} \\
& +V_{\mathrm{S}}[1+e(\omega, t)] \frac{\partial p_{\mathrm{SYS}}(\omega, t)}{\partial \omega},
\end{aligned}
$$

where $V_{\mathrm{S}}$ is the total solid volume, and $e$ is the void ratio. Then the total mechanical work received by sludge over time $[0, t]$ is

$$
E_{\mathrm{V}}=V_{\mathrm{S}} \int_{0}^{\omega_{\mathrm{S}}}\left[p_{\mathrm{SYS}}\left(\frac{\partial e}{\partial \omega}\right)+(1+e)\left(\frac{\partial p_{\mathrm{SYS}}}{\partial \omega}\right)\right] \mathrm{d} \omega .
$$

The lost work during a dewatering test, $\left(E_{\mathrm{V}}-\left(E_{\mathrm{F}}+E_{\mathrm{B}}\right)\right)$, is a result of the irreversible processes other than the intra-cake friction, and the friction between cake and device's wall (Zhao et al., 2003a, b), filter medium loss, or expansion work by water at the drainage port. The ratio $\left(E_{\mathrm{F}}+E_{\mathrm{B}}\right) / E_{\mathrm{V}}(=\eta)$ is the energy efficiency for dewatering the sludge based on the total work received by the sludge. The work done by the surroundings is $E_{\mathrm{V}} / \eta_{\text {ave }}$ (Eq. (1)) and the overall energy efficiency by the surroundings to suspension dewatering is $\left(E_{\mathrm{F}}+E_{\mathrm{B}}\right) / \int P \mathrm{~d} t=\eta \eta_{\mathrm{ave}}$. In the next sections methods for evaluating $E_{\mathrm{V}}, E_{\mathrm{B}}$ and $E_{\mathrm{F}}$ are addressed.

\subsection{Bond strength $E_{\mathrm{B}}$}

The bond strength between water and solid phase, $H_{\mathrm{B}}$, can be estimated by the TGA/DTA scheme proposed by Chen et al. (1997). Its procedures are briefly summarized as follows: The TGA and DTA tests, which are conducted simultaneously, evaluate, respectively, the heat flow $Q$ and the mass loss rate $(m)$ of a sample. Under thermal equilibrium conditions, specific enthalpy applied in water evaporation can be evaluated with $(\Delta H=Q / m, \mathrm{~kJ} / \mathrm{kg})$. As $\Delta H=\Delta H_{\mathrm{fg}}$, the standard enthalpy change for the bulk water, the evaporated water shows the same energy level as bulk water. According to Tsang and Vesilind (1990), this water is the non-bound, or free water, in the sample. If, conversely, $\Delta H>\Delta H_{\mathrm{fg}}$, the difference, $\Delta H-\Delta H_{\mathrm{fg}}$, can be attributed to the existence of the solid phase of the sample, defined as the bond strength of the water $\left(H_{\mathrm{B}}, \mathrm{kJ} / \mathrm{kg}\right)$. Water exhibiting increased bond strength requires high enthalpy for its separation from the solid surface. The total energy, then, required to break down the bond strength of a slurry to water content of $W$ is

$E_{\mathrm{B}}=V_{\mathrm{S}} \rho_{\mathrm{S}} \int_{\infty}^{W}\left(-H_{\mathrm{B}}\right) \mathrm{d} W$, where $W$ is residual water in the remaining slurry (cake and suspension) per unit solid mass. By this definition the moisture content $W$ approaches infinity for all diluted suspension, and is zero for completely dehydrated cake.

\subsection{Friction loss $E_{\mathrm{F}}$}

Friction loss owing to fluid outflow from the slurry is denoted as $E_{\mathrm{F}}$. Drag force acting on the fluid passing through solid volume $\mathrm{d} \omega$ can be stated as

$\mathrm{d} F_{\mathrm{F}}=A_{\mathrm{SYS}}\left(\frac{\mu}{K} q_{\mathrm{L}}\right) \mathrm{d} \omega$,

where $A_{\text {SYS }}$ is the filter area, $\mu$ is the filtrate viscosity, $K$ the local permeability of the cake, and $q_{\mathrm{L}}$ is the internal flow rate. Therefore, the energy loss attributable to the friction loss can be estimated as

$\frac{\mathrm{d} E_{\mathrm{F}}}{\mathrm{d} \omega}=H_{\mathrm{F}}=A_{\mathrm{SYS}} \int_{0}^{\omega_{\mathrm{S}}}\left[\frac{\mu}{K} q_{\mathrm{L}}\right] \mathrm{d} \omega$

or as an equivalent (Chu and Lee, 2002):

$E_{\mathrm{F}}=A_{\mathrm{SYS}} \int_{0}^{\omega_{\mathrm{S}}} \int_{0}^{\omega_{\mathrm{S}}}\left(\frac{\mu}{K} q_{\mathrm{L}}\right) \mathrm{d} \omega \mathrm{d} \omega$.

As stated, TGA/DTA tests can evaluate $H_{\mathrm{B}}$, with the reference state $\left(E_{\mathrm{B}}=0\right)$ at $W \rightarrow \infty$ (free water has no bond strength with solid surfaces). To evaluate $H_{\mathrm{V}}$ and $H_{\mathrm{F}}$, the reference state was chosen as $E_{\mathrm{V}}=E_{\mathrm{F}}=0$ at $\omega_{\mathrm{S}}=0$ (no cake limit) corresponding to the initial water content value of slurry. To evaluate $E_{\mathrm{V}}$ and $E_{\mathrm{F}}$ requires information on how the cake will deform $\left(p_{\mathrm{SYS}}-e\right.$ relation in Eq. (4)) and how the fluid will flow through $(K-\omega$ relation in Eq. (8)) the stressed slurry, respectively. The $p_{\mathrm{SYS}}-e$ relation and $K-\omega$ relation for centrifugation and consolidation tests are separately discussed as the following demonstrative examples.

\subsection{Centrifugation}

During centrifugal dewatering, the centrifugal force $\left(p_{\text {Cent }}\right)$ represents the system pressure $\left(p_{\text {SYS }}\right)$. Assuming that the cake thickness in the centrifuge is thin, $\varepsilon_{\mathrm{av}}$ can be used to represent the porosity distribution in the centrifugated cake. Considering the radii of various interfaces that developed in the centrifugated sludge, $r_{\mathrm{gl}}$, $r_{\mathrm{ls}}, r_{\mathrm{sc}}$, and $r_{\mathrm{cm}}$ represent air-supernatant interface, supernatant-suspension interface, suspension-cake interface, and cake--media interface, respectively (Chu and Lee, 2002). Then

$E_{\mathrm{V}}=\frac{A_{\mathrm{SYS}} \Omega^{2}}{2}\left[G_{1}(t)+G_{2}(t)\right]$

where $G_{1}(t)$ and $G_{2}(t)$ are functions of rotational radii that change over time (Appendix A), and presents the surface area of the centrifuge to sludge dewatering. 
Energy consumption increases with the rotational speed. The differentiation of $E_{\mathrm{V}}$ with respect to $W$ gives the specific $p-V$ work, $H_{\mathrm{V}}$.

With the centrate flow rate $\left(q_{\mathrm{L}}(t)\right)$ obtained and interface positions noted in the centrifugal test, $E_{\mathrm{F}}$ can be estimated as (Chu and Lee, 2002)

$E_{\mathrm{F}}=A_{\mathrm{SYS}} \mu \rho_{\mathrm{S}} \alpha_{\mathrm{av}}\left(1-\varepsilon_{\mathrm{av}}\right) q_{\mathrm{L}}\left(r_{\mathrm{cm}}-r_{\mathrm{sc}}\right)^{2}$,

where $\alpha_{\mathrm{av}}$ is the average specific resistance of the centrifugated cake. Based on this model, if only the interface positions, $r_{\mathrm{gl}}, r_{\mathrm{ls}}, r_{\mathrm{sc}}$, and $r_{\mathrm{cm}}$, can be tracked with the filtrate flow rate, then $E_{\mathrm{V}}$ and $E_{\mathrm{F}}$ can be subsequently estimated.

\subsection{Consolidation}

During sludge consolidation, the solid pressure $\left(p_{\mathrm{S}}\right)$ describes the system pressure $p_{\mathrm{SYS}}$. During the consolidation test, pressure drop in the cake was substantial and, hence, the detailed $p_{\mathrm{S}}-e$ relation (rheological behavior) for stressed cake is required. Lee and Wang (2000) summarized numerous correlations for the $p_{\mathrm{S}}-e$ relations proposed in the literature. Since the parameters in the various proposed correlations are determined by data fitting, adopting different forms of correlations of $p_{\mathrm{S}}-e$ relation does not significantly impact the predictions of the cake consolidation behavior.

By considering the cake as a visco-elastic object and adopting the analytical model proposed by Shirato et al. (1974), the expression of $E_{\mathrm{V}}$ can be stated as

$E_{\mathrm{V}}=C_{1} p_{\mathrm{Expr}}^{2}-C_{2}\left(1+e_{1}\right) p_{\mathrm{Expr}}$.

The functions $C_{1}$ and $C_{2}$ are functions of dewatering time (Appendix $\mathrm{B}$ ), and $e_{1}$ is the void ratio of the suspension before dewatering. It is clear that the energy consumed by consolidation increases with consolidation pressure.

Furthermore, friction drag loss can be stated using the Darcy's law as

$$
\begin{aligned}
E_{\mathrm{F}} & =A_{\mathrm{SYS}} \int_{0}^{\omega_{\mathrm{S}}} \int_{0}^{\omega_{\mathrm{S}}}\left(-\frac{\partial p_{\mathrm{L}}}{\partial \omega}\right) \mathrm{d} \omega \mathrm{d} \omega, \\
& =A_{\mathrm{SYS}} \int_{0}^{\omega_{\mathrm{S}}} \int_{0}^{\omega_{\mathrm{S}}}\left(\frac{\partial p_{\mathrm{S}}}{\partial \omega}\right) \mathrm{d} \omega \mathrm{d} \omega .
\end{aligned}
$$

Or equivalently,

$$
H_{\mathrm{F}}=\frac{\mathrm{d} E_{\mathrm{F}}}{\mathrm{d} \omega_{\mathrm{S}}}=\frac{p_{\mathrm{Expr}} A_{\mathrm{SYS}} C_{2}}{V_{\mathrm{S}}} .
$$

All parameters in Eqs. (9-12) can be estimated by fitting dewatering data to consolidation dewatering. Experiments described in Section 3 demonstrate the use of these equations to estimate energy consumption during sludge dewatering.

\section{Experimental}

\subsection{Sample}

An activated sludge sample taken from the wastewater treatment plant of Neili Bread Plant, Presidential Enterprise Co., Taoyuan, Taiwan was tested within $2 \mathrm{~h}$ after sampling. The COD and SS data of the supernatant drawn from the sludge, measured via EPA Standard Methods, were 22.6 and $14.3 \mathrm{mgl}^{-1}$, respectively. The weight percentage of dried solid of sludge, determined by weighing and drying at $102{ }^{\circ} \mathrm{C}$, was $0.83 \% \mathrm{w} / \mathrm{w}$.

Cationic polyelectrolyte, polymer T-3052, was obtained from Kai-Guan Inc., Taiwan. The polymer T3052 is a polyacrylamide with an average molecular weight of $10^{7}$, and a charge density of $20 \%$. The original activated sludge was employed in flocculation. Sample sludge was first put into the mixing vessel. The polymer solution was then gradually poured into the mixing vessel by stirring at $200 \mathrm{rpm}$ for $5 \mathrm{mins}$ followed by $50 \mathrm{rpm}$ for another $20 \mathrm{mins}$. The zeta potential for the original sample was negative, and was neutralized at $60 \mathrm{mgl}^{-1}$ (Zeter-Meter System 3.0, Zeter-Meter Inc., USA).

\subsection{TGA/DTA test}

Details for the TGA/DTA tests that can be found in Chen et al. (1997) are herein briefly summarized for the sake of completeness. The thermal analyzer (SETARAM, 77A-92) was employed to record thermographs with argon (Ar) utilized as the carrying gas, in which the TGA and DTA tests were performed simultaneously. From the DTA peak, energy flow rate into the sample cell can be estimated after calibration. The weight and time data represent the rate change of sample weight.

The sample was first vacuum filtered to remove some free water. Next, for the sake of uniformity, the resulting filter cake was completely blended, from which the sludge sample was randomly taken. Under each condition, three independent tests were performed and their averages reported. The sample employed was limited to approximately $10 \mathrm{mg}$ to minimize possible effects of non-uniform temperature distribution and mass transfer resistance within the sample body. Shie et al. (2004) utilized the same TGA test adopted herein and noted that the intrinsic chemical kinetics of sample pyrolysis could be realized with an initial sample mass of around $10 \mathrm{mg}$. The mass transfer limitation hence plays no significant role for the TGA test conducted herein. The temperature was raised from room temperature to a fixed temperature of $80^{\circ} \mathrm{C}$. With TGA and DTA data, the bond strength versus residual water content was calculated. 


\subsection{Dewatering test}

The arm-suspension centrifuge proposed by $\mathrm{Chu}$ and Lee (2001) was employed in the centrifugal tests. This centrifuge facilitates direct observations on the filtrate amounts and cake thickness under the centrifugal field, and, hence, provides rheological information of the centrifugated cake under stress. Rotational speed ranged between 400 and $1000 \mathrm{rpm}$, with an acceleration at the filter medium of $32-200 \mathrm{~g}$.

A constant head piston press (Triton Electronics Ltd., type 147) was employed in the consolidation tests. The sludge was placed in a stainless steel cylinder, $7.62 \mathrm{~cm}$ in diameter and $20 \mathrm{~cm}$ high. A hydraulic pressure of 1400-3000 psi was exerted on the piston to force water out of the sludge sample. Time evolution of the filtrate weight was then automatically recorded with an electronic balance connected to a personal computer. Given these data and solid density, time evolution for cake porosity was subsequently obtained. Chang and Lee (1998) provided the details for the consolidation experiment.

\section{Results and discussion}

\subsection{TGA/DTA test}

Fig. 2a shows the TGA/DTA results for the flocculated $(40 \mathrm{~g} / \mathrm{kg})$ sludge sample. The sample weight (TGA), rate of change (DTG), thermocouple output voltage (DTA), and reference cell temperature $\left(T_{\text {cell }}\right)$ were demonstrated to be functions of time. The sample mass decreased as the cell temperature increased, signaling the loss of water in response to input thermal energy. A period of near-constant DTG existed, corresponding to the amount of free water in the sludge. The falling-rate period followed the near-constant DTG period. Water removed during the falling-rate period exhibited an increased resistance to evaporation.

Fig. 2b shows $H_{\mathrm{B}}$ versus residual water $W$ curves for the sludge samples. The residual water content $(W)$ was directly calculated from the TGA data. Also, the $H_{\mathrm{B}}$ increased when the residual water content decreased. At $W>30 \mathrm{~kg} \mathrm{~kg}^{-1} \mathrm{DS}$, the bond strength was close to $1 \mathrm{~kJ} /$ $\mathrm{kg}$ (Herwijn et al., 1992). When the water content was less than $0.5 \mathrm{~kg} \mathrm{~kg}^{-1} \mathrm{DS}$, the bond strength exceeded $1000 \mathrm{~kJ} / \mathrm{kg}$, and was significantly close to that for chemisorption/chemical reactions.

At a $25-\mathrm{mg}^{-1}$ dose, the optimal dose of the sludge identified using filtration test (shown later), $H_{\mathrm{B}}$ for $W<4 \mathrm{~kg} \mathrm{~kg}^{-1} \mathrm{DS}$ was reduced, indicating that the adjacent water was loosened and became easier to be removed by flocculation. An overdose at $40 \mathrm{mgl}^{-1}$ of polymer also reduced $H_{\mathrm{B}}$ for $W>2.6 \mathrm{~kg} \mathrm{~kg}^{-1} \mathrm{DS}$ compared to the original sludge. But the polymer did
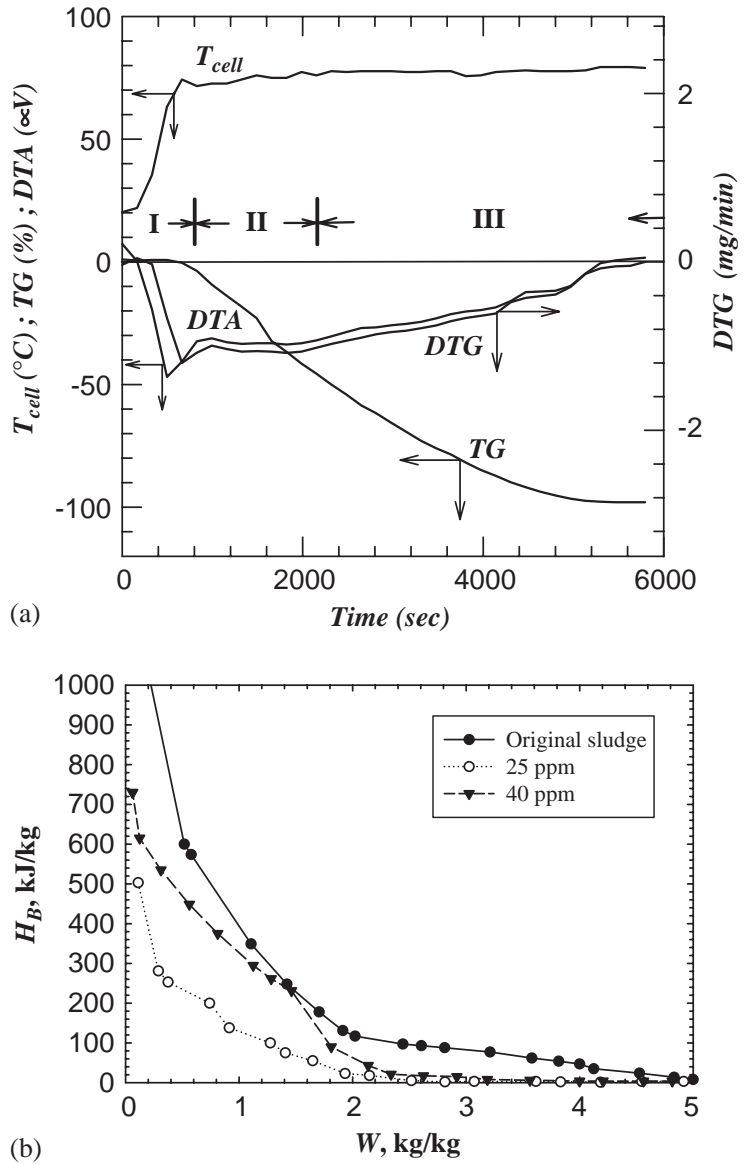

Fig. 2. (a) The TGA/DTA test for wastewater sludge $(40 \mathrm{~g} / \mathrm{kg}$ DS) and (b) the bond energy versus residue water in wastewater sludge, original and flocculated.

not significantly lower the bond strength for $W<2 \mathrm{~kg} \mathrm{~kg}^{-1} \mathrm{DS}$. Restated, an overdose of polymer would facilitate the release of some of the "easily removed" water, but could not release the tightly bound fraction of water. This result might be partly attributable to the adsorption of water on the excess flocculant molecules (Chu and Lee, 1999).

The $H_{\mathrm{B}}$ curves, as shown in Fig. $2 \mathrm{~b}$, presented the bond strength required to "release" water from solid surfaces in the tested sludge. Based on this curve, the energy required to release $1 \mathrm{~kg}$ of water from a sludge with a water content of $4 \mathrm{~kg} \mathrm{~kg}^{-1}$ DS to a "free-water" state was roughly $60 \mathrm{~kJ}$ for the original sludge and less than $4 \mathrm{~kJ}$ for the $25-\mathrm{mg}^{-1}$-flocculated sludge. Further dewatering to remove $1 \mathrm{~kg}$ of water from the sludge with a water content of $2 \mathrm{~kg} \mathrm{~kg}^{-1} \mathrm{DS}$ required $140 \mathrm{~kJ}$ for the original and $20 \mathrm{~kJ}$ for the $25-\mathrm{mg} \mathrm{l}^{-1}$-flocculated sludges. Further dewatering consumed more energy than the initial dewatering stage. 


\subsection{Centrifugal dewatering}

The centrifugal rate was low for the original sludge at 400-rpm centrifugation, as shown in Fig. 3a. Increasing the centrifugal acceleration or adding polyelectrolyte facilitated the dewatering rate. The dose of $25 \mathrm{mgl}^{-1}$ provided the best dewatering rate, while an overdose of $40 \mathrm{mgl}^{-1}$ reduced the dewatering rate (Fig. $3 \mathrm{~b}$ ). The optimal dose for the present sludge was $25 \mathrm{mgl}^{-1}$ in the centrifugal test, as mentioned in preceding sections.

Fig. 4 shows the $H_{\mathrm{V}}$ data during centrifugation with polyelectrolyte dose and centrifugal speed parameters. The work by the centrifuge on the sludge increased as centrifugal acceleration increased, and, thereby, water content decreased. Also, at the optimal dose of $25 \mathrm{mgl}^{-1}$, as identified in Fig. 4b, the $H_{\mathrm{V}}$ was lower than under- or overdosed regime at high $W$, and was higher than the other two at low $W\left(<60 \mathrm{~kg} \mathrm{~kg}^{-1} \mathrm{DS}\right)$. External work by the centrifuge to remove $1 \mathrm{~kg}$ of water from the sludge was comparably low, ca. $1 \mathrm{~kJ} / \mathrm{kg}$ at $400 \mathrm{rpm}$ and $5-9 \mathrm{~kJ} / \mathrm{kg}$ at $1000 \mathrm{rpm}$.

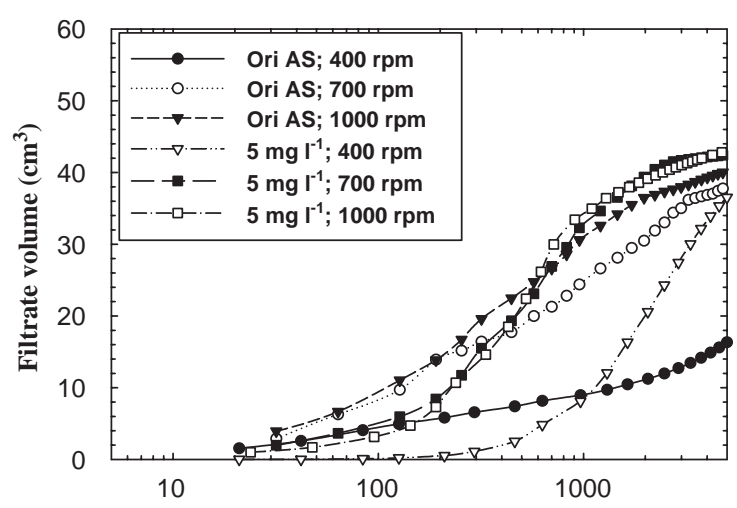

(a)

time (s)

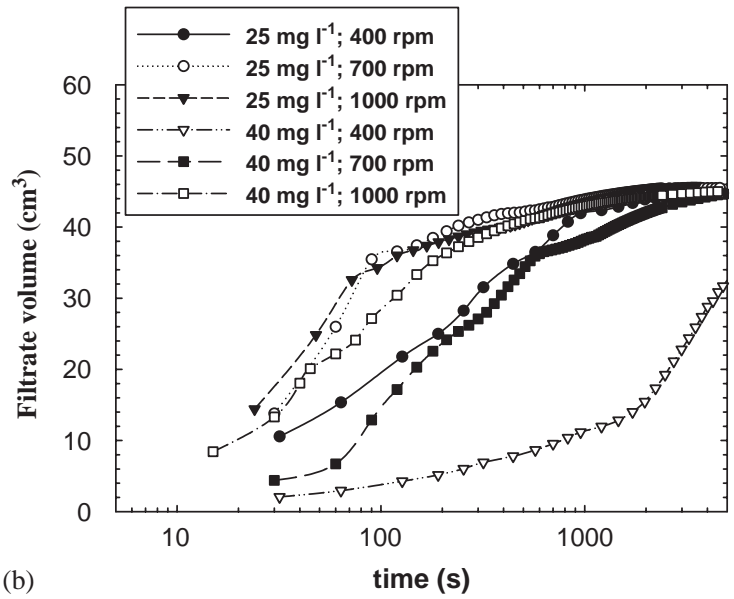

Fig. 3. Dewatering curves of centrifugated sludge: (a) original and $5-\mathrm{mg}^{-1}$ flocculated and (b) $25-$ and $40-\mathrm{mg}^{-1}$ flocculated.
The $E_{\mathrm{F}}$ data calculated accounted for less than $5 \%$ of $E_{\mathrm{V}}$, indicating that most of the friction loss did not occur in line with the intra-cake flow. These data were not shown here for the sake of brevity.

\subsection{Consolidation dewatering}

Fig. 5 illustrates the consolidation curves. The original sludge had a low consolidation rate. To remove $280 \mathrm{~g}$ of filtrate from the original sludge required $1000 \mathrm{~s}$, while the dose of $5 \mathrm{mgl}^{-1}$ polyelectrolyte reduced the corresponding consolidation time to $120-160 \mathrm{~s}$, depending on the consolidation pressure. A further increase in dosage to $25 \mathrm{mg} \mathrm{l}^{-1}$ demonstrated the best dewatering result, around $30 \mathrm{~s}$ to remove $280 \mathrm{~g}$ of filtrate at $3000 \mathrm{psi}$. An overdose of $40 \mathrm{mg}^{-1}$ polyelectrolyte yielded a slightly reduced dewatering rate. The optimal dose identified in the consolidation test corresponded to that in the centrifugal test $\left(25 \mathrm{mg} \mathrm{l}^{-1}\right)$.

Fig. 6 shows the $E_{\mathrm{V}}$ data under consolidation, with the polyelectrolyte dose and the consolidation pressure applied as parameters. The external work performed by the piston increased with increased consolidation pressure and with decreased water content. Also, at the optimal dosage of $25 \mathrm{mg}^{-1}$, as identified in Fig. 5b, the $H_{\mathrm{V}}$ was lower than the under- or overdosed regime at $W=1.7-9.0 \mathrm{~kg} \mathrm{~kg}^{-1} \mathrm{DS}$. This experimental result did not correspond to that in centrifugation test (Fig. 4). At $25-\mathrm{mgl}^{-1}$ dosage and consolidation pressure of 2200 psi, external work performed by the consolidation tester was not significant until $W=3.2 \mathrm{~kg} \mathrm{~kg}^{-1} \mathrm{DS}$. The 25- $\mathrm{mg}^{-1}$-flocculated sludge was, hence, easily dewatered to a residual water of $3.2 \mathrm{~kg} \mathrm{~kg}^{-1}$ using consolidation. The original sludge, on the other hand, required external work greater than $100 \mathrm{~kJ}$ to dewater the sludge at $2200 \mathrm{psi}$ to a water content of $4.2 \mathrm{~kg} \mathrm{~kg}^{-1} \mathrm{DS}$. The current flocculation conditioning effectively enhanced the sludge dewaterability by reducing the bond strength between water and the solid phase.

The estimated $H_{\mathrm{F}}$ data were generally lower than $10 \%$ of $H_{\mathrm{V}}$ and were, thereby, not shown in the figures for sake of simplicity.

\section{Discussion}

$H_{\mathrm{V}} \gg H_{\mathrm{F}}$ as demonstrated in the current centrifugation and consolidation tests. The resistance of internal flow through filter cake did not dominate.

The $H_{\mathrm{B}}$ curves were also plotted on Figs. 4 and 6 for comparison. During the initial stage of centrifugal dewatering, water content was high, and corresponded to low $H_{\mathrm{B}}$. Restated, the adjacent water is easily removed from the solid surface at this moisture content level. The $H_{\mathrm{V}}$ provided by the centrifuge was even lower. Most energy input was therefore utilized to overcome 


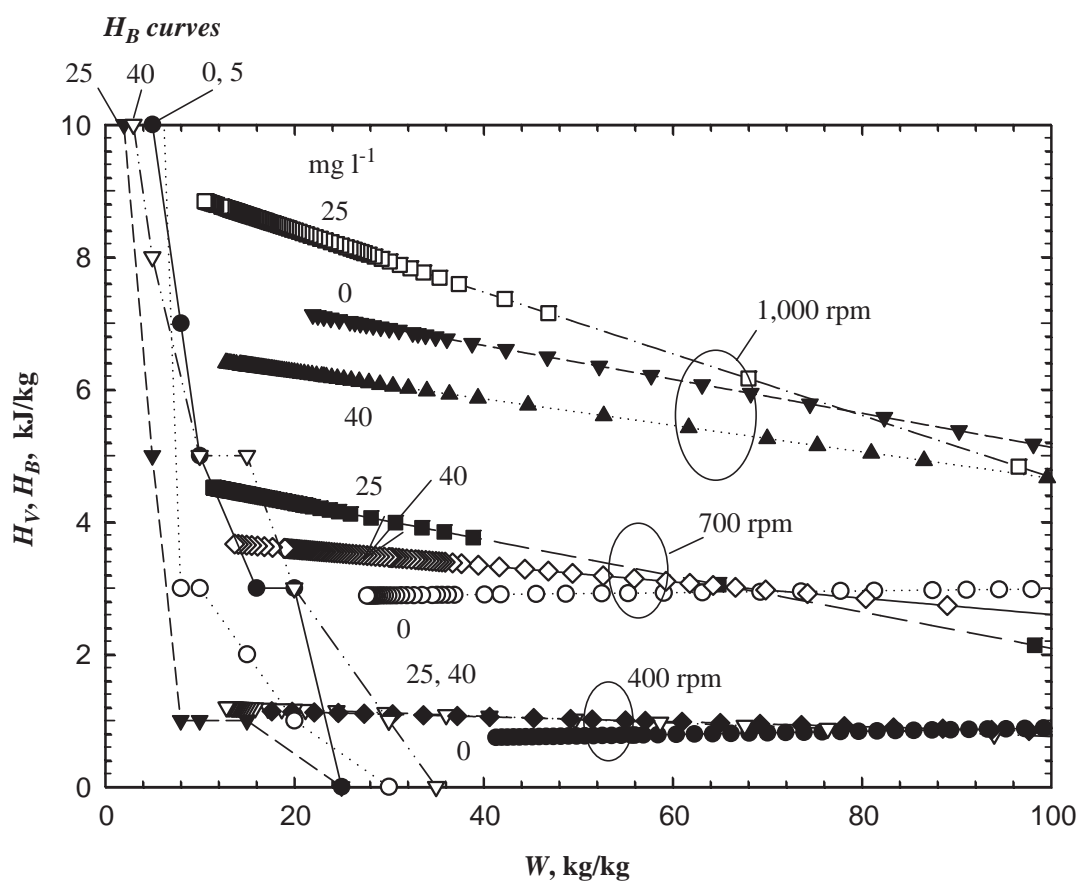

Fig. 4. The $H_{\mathrm{V}}$ input during centrifugation and the $H_{\mathrm{B}}$ curves of sludges.
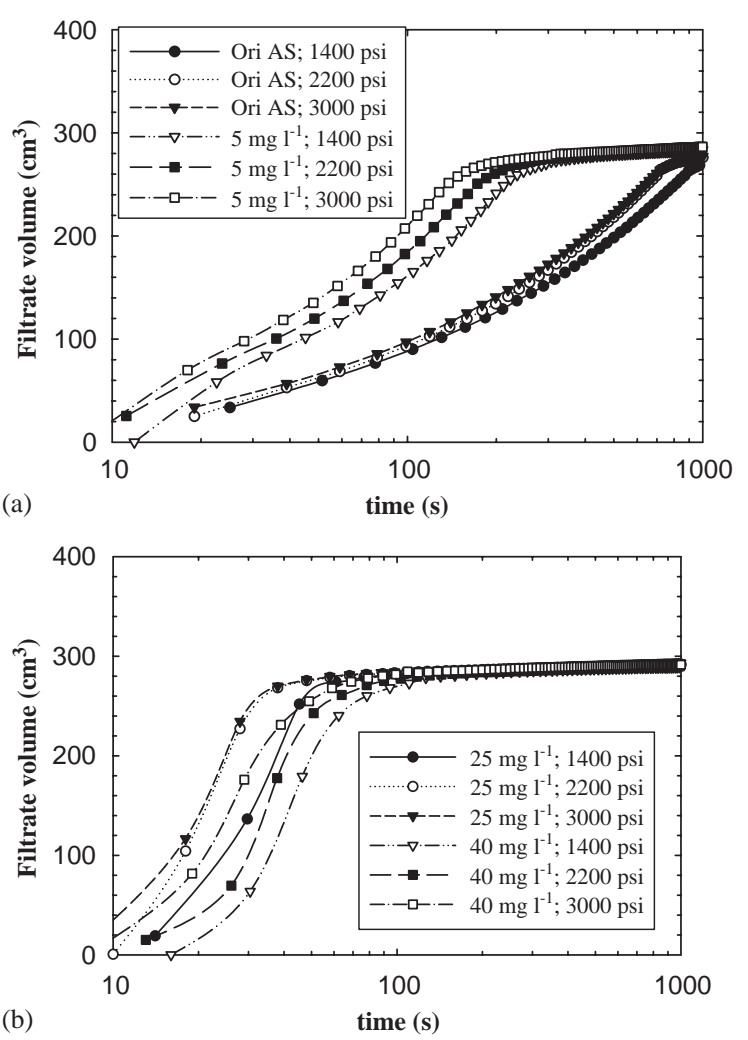

Fig. 5. Dewatering curves of consolidated sludge: (a) original and $5-\mathrm{mg}^{-1}$ flocculated and (b) $25-$ and $40-\mathrm{mg}^{-1}$ flocculated. process irreversibility other than intra-cake friction. The dewatering rate was, thereby, not correlated with the $H_{\mathrm{B}}-W$ curve until the latter was reduced to less than $20-30 \mathrm{~kg} \mathrm{~kg}^{-1} \mathrm{DS}$. At high rotational speeds or at the optimal dose of flocculant, $H_{\mathrm{V}}$ was high, indicating that the process was highly irreversible. This experimental result was likely due to the deterioration of the welldeveloped network structure of optimally flocculated sludge cake under the centrifugal field. Releasing adjacent water from solid surfaces did not affect the dewatering rate under this scenario. With a low $W$ regime, $H_{\mathrm{B}}$ increased rapidly during dewatering. Since the $H_{\mathrm{V}}-W$ curves exhibited a lower slope than the $H_{\mathrm{B}}-W$ curve, dewatering ceased at $H_{\mathrm{V}}=H_{\mathrm{B}}$. Moreover, although the dewatering rate was quickest with optimal dose or with the fastest rotational speed (Fig. 3), the corresponding energy demand is maximized. Consequently, a compromise between process time and energy demand is needed.

The current consolidation tester can provide a much higher $H_{\mathrm{V}}$ on sludge than the tested centrifuge. Depending on the polyelectrolyte dose, the $H_{\mathrm{B}}$ was close to $H_{\mathrm{V}}$ until a critical water content level was reached, then $H_{\mathrm{V}}>H_{\mathrm{B}}$. For instance, the $H_{\mathrm{V}}$ for consolidating original sludge at 3000 psi was close to $H_{\mathrm{B}}$ up to $W=4 \mathrm{~kg} \mathrm{~kg}^{-1} \mathrm{DS}$, or at $1400 \mathrm{psi}$ up to $W=7 \mathrm{~kg} \mathrm{~kg}^{-1} \mathrm{DS}$. Therefore, most energy consumed during consolidation test was utilized to release adjacent water from solid surfaces since the $H_{\mathrm{B}}$ is high at low- $W$ regime. Other process irreversibility was relatively small. 

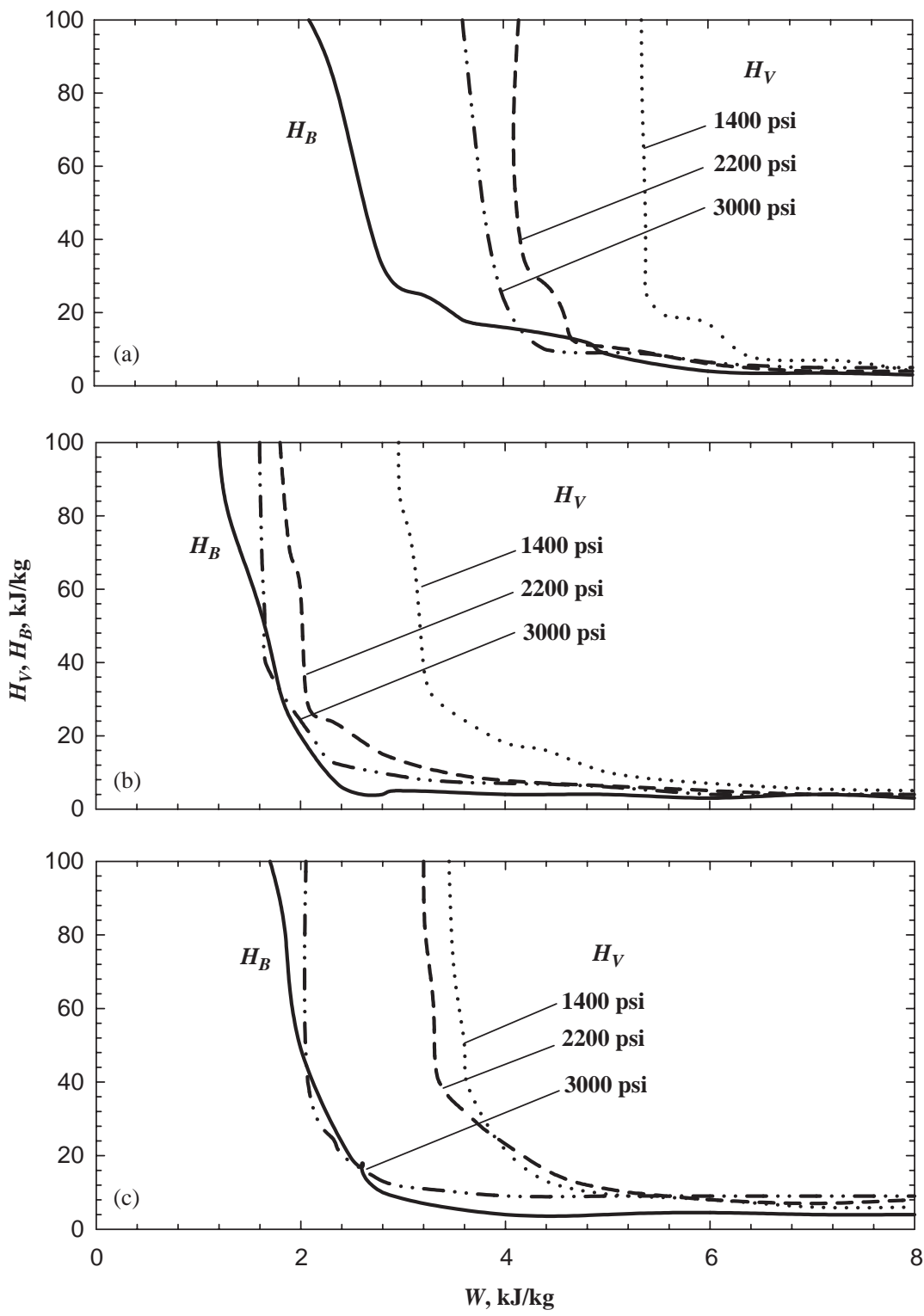

Fig. 6. The $H_{\mathrm{V}}$ input during consolidation and the $H_{\mathrm{B}}$ (bold) curves of sludges: (a) original sludge, (b) 25-ppm sludge and (c) 40-ppm sludge.

When $W<4 \mathrm{~kg} \mathrm{~kg}^{-1} \mathrm{DS}$ at $3000 \mathrm{psi}$ or $W<7 \mathrm{~kg} \mathrm{~kg}^{-1} \mathrm{DS}$ at $1400 \mathrm{psi}, H_{\mathrm{V}}$ became higher than $H_{\mathrm{B}}$. The majority of energy consumed was applied to overcome internal frictions and possible particle re-orientation in the cake. The $H_{\mathrm{V}}$ had become so large that further dewatering through mechanical means below $W=3.4 \mathrm{~kg} \mathrm{~kg}^{-1} \mathrm{DS}$ was practically impossible. Lee and Hsu (1995) regarded this water content as the bound water identified by adopting the consolidation test. The $H_{\mathrm{V}}$ (or $H_{\mathrm{B}}$ ) versus $W$ curves for flocculated sludge resembled, in character, those for original sludge. Just, as $H_{\mathrm{B}}$ was reduced at an optimal dosage $\left(25 \mathrm{mgl}^{-1}\right)$, the corresponding $H_{\mathrm{V}}$ curves in Fig. 7 shifted leftward to the low- $W$ regime. The $H_{\mathrm{V}}-W$ curves followed closely the $H_{\mathrm{B}}-W$ curve at $W>1.9$ or $>5.5 \mathrm{~kg} \mathrm{~kg}^{-1}$ DS under 3000 or $1400 \mathrm{psi}$, respectively. The critical amount of water reduced at the optimal dosage, indicating that the cake became dryer when the consolidation reached mechanical equilibrium.

The ratio of $\left(\left(E_{\mathrm{F}}+E_{\mathrm{B}}\right) / E_{\mathrm{V}}\right)$ estimated how far the system deviated from an "ideal" dewatering system. Fig. 7 presents this deviation based on the data shown in Figs. 4 and 6. If this ratio approaches unity, the energy 


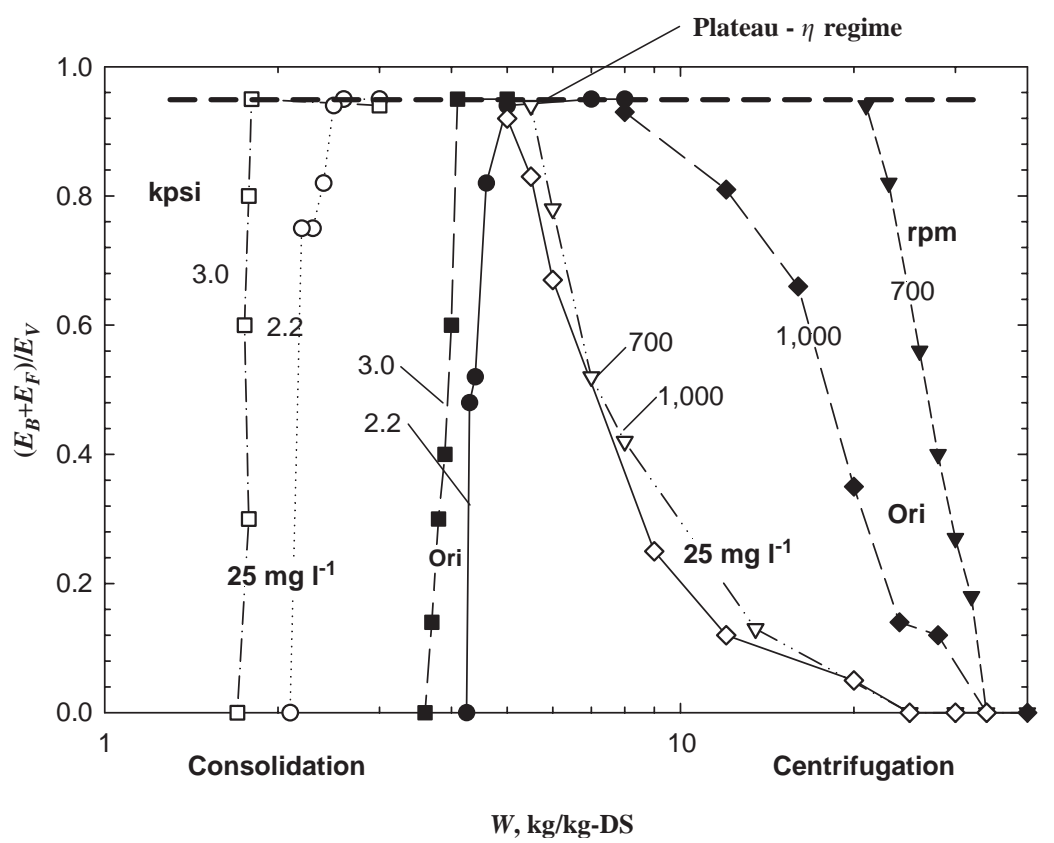

Fig. 7. Ratio $\left(E_{\mathrm{B}}+E_{\mathrm{F}}\right) / E_{\mathrm{V}}$ versus $W$ curves. Original and flocculated $\left(25 \mathrm{mgl}^{-1}\right)$ sludge samples.

consumption rate $\left(E_{\mathrm{V}}\right)$ is close to the minimum energy demand $\left(E_{\mathrm{B}}+E_{\mathrm{F}}\right)$, and hence the energy efficiency to dewatering is high. Centrifugation tests confirmed an increasing ratio while $W$ was reducing. Consolidation tests exhibited a decreasing ratio with low $W$ values. Also, the curves shown in Fig. 7 shifted left after flocculation, or increased consolidated pressure/centrifugal acceleration. The energy efficiency for dewatering depended on dewatering methods, dewatering pressure, and the state of flocculation. Regardless of the applied dewatering processes, a plateau regime of efficiency of around $95 \%$ existed on the ratio versus $W$ curves. Initially, the average solid content was low (large $W$ ). The fast dewatering rate through the filter media and the deterioration of cake structure under stress consumed most of the input energy, resulting in a low-energy efficiency. Since during the final stage the cake was highly compressible and had been satisfactorily compressed markedly close to filter media (Lee et al., 2000), the water that had been removed experienced marked resistance through the skin layer, and again yielded lowenergy efficiency. The energy economy could be maximized to operate closely at the high- $\eta$ plateau regime.

\section{Conclusions}

This work examined the minimum amount of mechanical work required to dewater a suspension, and considered the total energy input received by the suspension from the dewatering device $\left(E_{\mathrm{V}}\right)$, the bond strength between the adjacent water and solid surfaces $\left(E_{\mathrm{B}}\right)$, and the intra-cake friction loss $\left(E_{\mathrm{F}}\right)$. To remove water, the bond strength was first broken, and then water was removed from the cake, thus providing an estimate of minimum work needed to dewater a sludge as $E_{\mathrm{B}}+E_{\mathrm{F}}$. An activated sludge was taken as the test sample. The bond strength of water was estimated with the thermal method proposed by Chen et al. (1997), with excess enthalpy employed to evaporate water to be as the bond strength. Friction loss was assessed using a modified Darcy's law, which required information on suspension rheology and dewatering rate as model parameters. The forms of $E_{\mathrm{V}}$ and $E_{\mathrm{F}}$ were evaluated by applying centrifugal dewatering and pressure consolidation as the demonstration examples.

During the preliminary stage of centrifugal dewatering, most of the energy input was used to overcome the process irreversibility other than the intra-cake friction, such as cake structure deterioration, that lead to lowenergy efficiency. Increasing the centrifugal speed or to flocculate at optimal dosage required high-energy input. Since the centrifuge could not provide sufficiently high levels of energy, dewatering ceased when the breakdown of bond strength became important in dewatering. During the consolidation test, most input energy was consumed in breaking down bond strength up to a critical residual water content. Further dehydration exceeding this critical water content required extremely high-energy input. To consolidate at higher pressure or 
to flocculate at optimal dosage yielded low critical water content levels. The ratio of $\left(\left(E_{\mathrm{F}}+E_{\mathrm{B}}\right) / E_{\mathrm{V}}\right)$ proved how far the system deviated from an "ideal" dewatering system. This ratio increased with decreasing residual water content during centrifugation, and, conversely decreased at consolidation. A plateau regime of highefficiency was noted in all tests in which the energy efficiency could be maximized.

\section{Acknowledgment}

This work was supported by the National Science Council, ROC.

\section{Appendix A. Derivation of Eq. (9)}

The centrifugal force acting on the sludge could be estimated as follows (Tiller and Hsyung, 1993):

$$
\begin{aligned}
p_{\mathrm{Cent}}=\Omega^{2} & {\left[\int_{r_{\mathrm{gl}}}^{r_{\mathrm{ls}}} \rho_{\mathrm{L}} r \mathrm{~d} r+\int_{r_{\mathrm{ls}}}^{r_{\mathrm{sc}}} \rho_{\text {Slurry }} r \mathrm{~d} r\right.} \\
& \left.+\int_{r_{\mathrm{sc}}}^{r_{\mathrm{cm}}}\left(\rho_{\mathrm{L}} r+\Delta \rho(1-\varepsilon) r\right) \mathrm{d} r\right],
\end{aligned}
$$

where $\Delta \rho$ is the density difference between the solid and the liquid, $\varepsilon$ is the local porosity of the wet cake, and $r_{\mathrm{gl}}, r_{\mathrm{ls}}, r_{\mathrm{sc}}$, and $r_{\mathrm{cm}}$ are the rotational radii of air-supernatant interface, supernatant-suspension interface, the suspension-cake interface, and the cake-media interface, respectively. Changing the coordinate system of Eq. (A.1) into a material coordinate $\omega$, we can reach the following equation:

$$
\begin{aligned}
\frac{\partial E_{\mathrm{V}}}{\partial r}= & p_{\mathrm{Cent}} \frac{\partial V}{\partial r}+V \frac{\partial p_{\mathrm{Cent}}}{\partial r} \\
= & {\left[\int_{0}^{r}\left(\frac{\partial p_{\mathrm{Cent}}}{\partial r}\right) \mathrm{d} r\right] A_{\mathrm{SYS}} } \\
& +A_{\mathrm{SYS}}\left(r_{\mathrm{cm}}-r_{\mathrm{gl}}\right)\left(\frac{\partial p_{\mathrm{Cent}}}{\partial r}\right) .
\end{aligned}
$$

The substitution of Eq. (A.1) into Eq. (A.2) yields

$E_{\mathrm{V}}=\frac{A_{\mathrm{SYS}} \Omega^{2}}{2}\left[G_{1}(t)+G_{2}(t)\right]$

where

\section{Appendix B. Derivation of Eq. (11)}

Shirato et al. (1974) analytically derived the distributions of pressure and void ratios in a consolidated cake as follows:

$p_{\mathrm{S}}+p_{\mathrm{L}}=p_{\mathrm{Expr}}$,

$p_{\mathrm{S}}=p_{\text {Expr }}\left\{1+\sin \left(\frac{\pi \omega}{2 \omega_{\mathrm{S}}}\right)\left[D_{1} \exp \left(h_{1} t\right)+D_{2} \exp \left(h_{2} t\right)\right]\right\}$,

$$
\begin{aligned}
e= & e_{1}-a_{\mathrm{E}} \int_{0}^{t}\left(\frac{\partial p_{\mathrm{S}}}{\partial t}\right) \mathrm{d} t-a_{\mathrm{C}} \eta_{\mathrm{c}} \int_{0}^{t}\left[p_{\mathrm{S}}(\omega, \tau)\right. \\
& \left.-p_{\mathrm{S} 1}\right] \exp \left[-\eta_{\mathrm{c}}(t-\tau)\right] \mathrm{d} \tau,
\end{aligned}
$$

where $p_{\text {Expr }}$ is the consolidation pressure and $e_{1}$ is the initial void ratio $(t=0)$ of suspension. Other parameters in Eqs. (B.2) and (B.3) are defined as follows:

$$
\varphi=\frac{B}{1-B}, \quad a_{\mathrm{E}}=\frac{1}{\mu \rho_{\mathrm{S}} \alpha_{\mathrm{av}} C_{\mathrm{e}}}, \quad a_{\mathrm{C}}=\phi a_{\mathrm{E}}, \quad(\mathrm{B} .4 \mathrm{a}-\mathrm{c})
$$$$
\begin{aligned}
& h_{1}=\frac{-\Psi}{2}-\frac{1}{2} \sqrt{\Psi^{2}-\frac{C_{\mathrm{e}} \eta_{\mathrm{c}} \pi^{2}}{\omega_{\mathrm{S}}^{2}}}, \\
& h_{2}=\frac{-\Psi}{2}+\frac{1}{2} \sqrt{\Psi^{2}-\frac{C_{\mathrm{e}} \eta_{\mathrm{c}} \pi^{2}}{\omega_{\mathrm{S}}^{2}}},
\end{aligned}
$$

$$
\Psi=\beta \eta_{\mathrm{c}}+\eta_{\mathrm{c}}+\frac{C_{\mathrm{e}} \pi^{2}}{4 \omega_{\mathrm{S}}^{2}}, \quad D_{1}=\frac{\pi C_{\mathrm{e}}\left(\eta_{\mathrm{c}}+h_{1}\right)^{2}}{4 \omega_{\mathrm{S}}^{2} h_{1}\left[\left(\eta_{\mathrm{c}}+h_{1}\right)^{2}+\beta \eta_{\mathrm{c}}^{2}\right]}
$$

$D_{2}=\frac{\pi^{2} C_{\mathrm{e}}\left(\eta_{\mathrm{c}}+h_{2}\right)^{2}}{4 \omega_{\mathrm{S}}^{2} h_{2}\left[\left(\eta_{\mathrm{c}}+h_{2}\right)^{2}+\beta \eta_{\mathrm{c}}^{2}\right]}$

where $p_{\mathrm{L}}$ is the liquid pressure, the $B$ the ratio of secondary consolidation to the total consolidation, $\eta_{\mathrm{c}}$ the creep factor, and $C_{\mathrm{e}}$ the consolidation coefficient (Chang and Lee, 1998).

Substituting Eqs. (B.1)-(B.4) into Eq. (4) leads to the following form of $E_{\mathrm{V}}$ :

$$
G_{1}=\frac{\rho_{\mathrm{L}}\left[2 r_{\mathrm{gl}}^{3}+r_{\mathrm{ls}}\left(r_{\mathrm{ls}}^{2}-3 r_{\mathrm{gl}}^{2}\right)\right]+\rho_{\mathrm{Slurry}}\left[2 r_{\mathrm{ls}}^{3}+r_{\mathrm{sc}}\left(r_{\mathrm{sc}}^{2}-3 r_{\mathrm{ls}}^{2}\right)\right]+\left(\rho_{\mathrm{L}}+\Delta \rho\left(1-\varepsilon_{\mathrm{av}}\right)\right)\left[2 r_{\mathrm{sc}}^{3}+r_{\mathrm{cm}}\left(r_{\mathrm{cm}}^{2}-3 r_{\mathrm{sc}}^{2}\right)\right]}{3}
$$

$$
\begin{aligned}
G_{2}= & \left(r_{\mathrm{cm}}-r_{\mathrm{gl}}\right)\left[\rho_{\mathrm{L}}\left(r_{\mathrm{ls}}^{2}-r_{\mathrm{gl}}^{2}\right)+\rho_{\text {Slurry }}\left(r_{\mathrm{sc}}^{2}-r_{\mathrm{ls}}^{2}\right)\right. \\
& \left.+\left(\rho_{\mathrm{L}}+\Delta \rho\left(1-\varepsilon_{\mathrm{av}}\right)\right)\left(r_{\mathrm{cm}}^{2}-r_{\mathrm{sc}}^{2}\right)\right],
\end{aligned}
$$

where $\varepsilon_{\mathrm{av}}$ is the average porosity in the centrifugated cake, and all interfaces were functions of time.

$$
\begin{aligned}
E_{\mathrm{V}}= & V_{\mathrm{S}}\left\{a_{\mathrm{E}} a_{\mathrm{C}} \eta_{\mathrm{c}} p_{\mathrm{Expr}}^{2} F_{1}\left[F_{2}+1\right]\left[F_{2}-D_{1}-D_{2}\right]\right\} \\
& \left.-\left(1+e_{1}\right) p_{\mathrm{Expr}} F_{2}\right\} \\
= & C_{1} p_{\mathrm{Expr}}^{2}-C_{2}\left(1+e_{1}\right) p_{\mathrm{Expr}}
\end{aligned}
$$


where

$$
\begin{aligned}
F_{1}= & \frac{1-\exp \left(-\eta_{\mathrm{c}} t\right)}{\eta_{\mathrm{c}}}+D_{1}\left[\frac{\exp \left(h_{1} t\right)-\exp \left(-\eta_{\mathrm{c}} t\right)}{h_{1}+\eta_{\mathrm{c}}}\right] \\
& +D_{2}\left[\frac{\exp \left(h_{2} t\right)-\exp \left(-\eta_{\mathrm{c}} t\right)}{h_{2}+\eta_{\mathrm{c}}}\right], \\
F_{2}= & D_{1} \exp \left(h_{1} t\right)+D_{2} \exp \left(h_{2} t\right), \\
C_{1}= & V_{\mathrm{S}} a_{\mathrm{E}} a_{\mathrm{C}} \eta_{\mathrm{c}} F_{1}\left[F_{2}+1\right]\left[F_{2}-D_{1}-D_{2}\right], \\
C_{2}= & V_{\mathrm{S}} F_{2} .
\end{aligned}
$$

\section{References}

Bejan, A., 1996. Entropy Generation Minimization. CRC Press, Boca Raton, FL.

Chang, I.L., Lee, D.J., 1998. Ternary expression stage of biological sludge dewatering. Water Res. 32, 905-914.

Chen, G.W., Chang, I.L., Hung, W.T., Lee, S.F., Lee, D.J., 1997. Continuous classification of moisture content in waste activated sludge. J. Environ. Eng. ASCE 123, 253-258.

Chu, C.P., Lee, D.J., 1999. Moisture distributions in sludges: effects of cationic polymer conditioning. J. Environ. Eng. ASCE 125, 340-345.

Chu, C.P., Lee, D.J., 2001. Experimental analysis of centrifugal dewatering process of polyelectrolyte flocculated waste activated sludge. Water Res. 35, 2377-2384.

Chu, C.P., Lee, D.J., 2002. Centrifugation dewatering of sludges. Dry. Tech. 20, 953-966.

Haschemeyer, A.E.M., DeVries, A.L., Guschibauer, W., 1977. Water bond by antifreeze glycoproteins from antarctic fish. Nature 269, 87-88.

Herwijn, A.J.M., Van Dijke, D.Q.A., La Heij, E.J., Coumans, W.J., Kerkhof, P.J.A.M., 1992. The solid-water bound strength in sewage sludge. AIChE Annual Meeting, Miami Beach, pp. 1073-1078.

Heukelekian, H., Weisberg, E., 1956. Bound water and activated sludge bulking. Sewage Ind. Wastes 28, 558-574.

Karr, P.R., Keinath, T.M., 1978. Influence of particle size on sludge dewaterability. J. Water Poll. Control Fed. 50, 1911-1930.

Katsiris, N., Kouzeli-Katsiri, A., 1987. Bound water content of biological sludge in relation to filtration and dewatering. Water Res. 21, 1319-1327.
Kopp, J., Dichtl, N., 2000. Prediction of full-scale dewatering results by determining the water distribution of sewage sludges. Water Sci. Tech. 42 (9), 141-149.

Kopp, J., Dichtl, N., 2001a. Prediction of full-scale dewatering results of sewage sludges by the physical water distribution. Water Sci. Tech. 43 (11), 135-143.

Kopp, J., Dichtl, N., 2001b. Influence of the free water content on the dewaterability of sewage sludges. Water Sci. Tech. 44 (10), 177-183.

Lee, D.J., Hsu, Y.H., 1995. Measurement of bound water in sludges: a comparative study. Water Environ. Res. 67, 310-317.

Lee, D.J., Wang, C.H., 2000. Theories of cake filtration and consolidation and implications to sludge dewatering. Water Res. 34, 1-20.

Lee, H.B., Jhon, M.S., Andrade, J.D., 1975. Nature of water in synthetic hydrogels. J. Colloid Interf. Sci. 51, 225-231.

Lee, D.J., Ju, S.P., Kwon, J.H., Tiller, F.M., 2000. Filtration of highly compactible filter cake: variable internal flow rate. AIChE J. 46, 110-118.

Lewicki, P.P., Busk, G.C., Labuza, T.P., 1978. Measurement of gel water-bond capacity of gelatin, potato starch, and carrageenan gels by suction pressure. J. Colloid Interf. Sci. 64, 501-509.

Robinson, J., Knocke, W.R., 1992. Use of dilatometric and drying techniques for assessing dewatering characteristics. Water Environ. Res. 64, 60-68.

Shie, J.L., Lin, J.P., Chang, C.Y., Wu, C.H., Lee, D.J., Chang, C.F., Chen, Y.H., 2004. Oxidative thermal treatment of oil sludge at low heating rates. Energy \& Fuels 18, 1272-1281.

Shirato, M., Murase, T., Tokunaga, A., Yamada, O., 1974. Calculations of consolidation period in expression operations. J. Chem. Eng. Jpn. 7, 229-231.

Tiller, F.M., Hsyung, N.B., 1993. Unifying the theory of thickening, filtration, and centrifugation. Water Sci. Tech. 28, 1-8.

Tsang, K.R., Vesilind, P.A., 1990. Moisture distribution in sludges. Water Sci. Tech. 22, 135-142.

Vesilind, P.A., 1994. The role of water in sludge dewatering. Water Environ. Res. 66, 4-11.

Zhao, J., Wang, C.H., Lee, D.J., Tien, C., 2003a. Plastic deformation in cake consolidation. J. Colloid Interf. Sci. $261,133-145$.

Zhao, J., Wang, C.H., Lee, D.J., Tien, C., 2003b. Cake consolidation in a compression-permeability cell: effect of side-wall friction. J. Colloid Interf. Sci. 262, 60-72. 